\title{
Quantum Gravitational Corrections to the Real Klein-Gordon Field in the Presence of a Minimal Length
}

\author{
S. K. Moayedi ${ }^{a *}$, M. R. Setare ${ }^{b \dagger}$ and H. Moayeri ${ }^{a \ddagger}$ \\ a Department of Physics, Arak University, 38156-879 Arak, Iran \\ ${ }^{b}$ Department of Science, Payame Noor University, Bijar, Iran
}

\begin{abstract}
The $(\mathrm{D}+1)$-dimensional $\left(\beta, \beta^{\prime}\right)$-two-parameter Lorentz-covariant deformed algebra introduced by Quesne and Tkachuk [C. Quesne and V. M. Tkachuk, J. Phys. A: Math. Gen. 39, 10909 (2006).], leads to a nonzero minimal uncertainty in position (minimal length). The Klein-Gordon equation in a $(3+1)$-dimensional space-time described by Quesne-Tkachuk Lorentz-covariant deformed algebra is studied in the case where $\beta^{\prime}=2 \beta$ up to first order over deformation parameter $\beta$. It is shown that the modified Klein-Gordon equation which contains fourth-order derivative of the wave function describes two massive particles with different masses. We have shown that physically acceptable mass states can only exist for $\beta<\frac{1}{8 m^{2} c^{2}}$ which leads to an isotropic minimal length in the interval $10^{-17} m<\left(\triangle X^{i}\right)_{0}<10^{-15} \mathrm{~m}$. Finally, we have shown that the above estimation of minimal length is in good agreement with the results obtained in previous investigations.
\end{abstract}

Keywords: Quantum gravity; Minimal length; Relativistic wave equations; Klein-Gordon equation

PACS numbers: 03.65.Pm; 04.60.-m

\section{Introduction}

One of the most important problems in theoretical physics is to unify general relativity and quantum mechanics. Together they are able to describe almost all known phenomena from

\footnotetext{
*E-mail: s-moayedi@araku.ac.ir

${ }^{\dagger}$ E-mail: rezakord@ipm.ir

${ }^{\ddagger}$ E-mail: h-moayeri@phd.araku.ac.ir
} 
the scale of subatomic particles all the way up to the rotations of galaxies and even the largescale structure of the universe. On the other hand, according to fundamental principles of quantum mechanics the position and momentum of a particle cannot be measured simultaneously. The uncertainties of position and momentum are related by Heisenberg's uncertainty relation $\Delta x \Delta p \geq \frac{\hbar}{2}$. An important consequence is that in order to probe arbitrarily small length-scales, one has to use probes of sufficiently high energy, and thus momentum. At present, there is theoretical evidence that in quantum gravity there might exist a minimal observable distance on the order of the Planck length, $l_{P}=\sqrt{\frac{G \hbar}{c^{3}}} \simeq 1.6 \times 10^{-35} \mathrm{~m}$, where $\mathrm{G}$ is the Newton's constant. From string theory we know that a string cannot probe distances smaller than its length. The existence of such a minimal observable length which is motivated from perturbative string theory, quantum gravity and black hole gedanken experiments, leads to a generalization of Heisenberg uncertainty principle. This generalized or gravitational uncertainty principle (GUP) can be written as

$$
\triangle X \triangle P \geq \frac{\hbar}{2}\left[1+\beta(\triangle P)^{2}+\ldots\right]
$$

where $\beta$ is a positive parameter [1-7]. It is clear that in Eq. (1), $\Delta X$ is always larger than $(\triangle X)_{\min }=\hbar \sqrt{\beta}$. Nowadays, physicists are trying to reformulate the quantum field theory in the presence of a minimal observable length and there is hoping that this approach causes unwanted divergencies can be eliminated or modified in quantum field theory [4].

Our paper is organized as follows. In Sec. 2, we will obtain a generalized Klein-Gordon equation of the fourth-order, describing particles with spin-0 based on the Lorentz-covariant deformed algebra with minimal length which was introduced by Quesne and Tkachuk in Refs. [2,3]. In Sec. 3, the solutions of the generalized wave equation for free motion of a Klein-Gordon particle have been obtained and it has been shown that these solutions are associated with two different mass states. Then, we will propose a direct method for obtaining the minimal length. We find that the minimal length is of the order of $10^{-16} \mathrm{~m}$. Our estimation of the minimal length is in good agreement with the results obtained in the papers [8-13]. The conclusions are presented in Sec. 4. Two appendices conclude this paper. Appendix A presents the Lagrangian formulation of the real Klein-Gordon field in the presence of a minimal length. In appendix B, the Feynman propagator in momentum space for the real Klein-Gordon field in the presence of a minimal length has been obtained and it has been shown that the generalized real Klein-Gordon field possesses one physical state with the mass $m_{-}$, and one Weyl ghost with the mass $m_{+}$. SI units are used throughout this paper.

\section{Generalized Klein-Gordon Equation}

In 2006, Quesne and Tkachuk introduced a Lorentz-covariant deformed algebra which describes a $(\mathrm{D}+1)$-dimensional quantized space-time $[2,3]$. This algebra in $(3+1)$-dimensional space-time is characterized by the following modified commutation relations

$$
\left[X^{\mu}, P^{\nu}\right]=-i \hbar\left(g^{\mu \nu}\left(1-\beta P_{\rho} P^{\rho}\right)-\beta^{\prime} P^{\mu} P^{\nu}\right)
$$




$$
\begin{gathered}
{\left[X^{\mu}, X^{\nu}\right]=i \hbar \frac{2 \beta-\beta^{\prime}-\left(2 \beta+\beta^{\prime}\right) \beta P_{\rho} P^{\rho}}{1-\beta P_{\rho} P^{\rho}}\left(P^{\mu} X^{\nu}-P^{\nu} X^{\mu}\right),} \\
{\left[P^{\mu}, P^{\nu}\right]=0,}
\end{gathered}
$$

where $\mu, \nu=0,1,2,3$ and $\beta, \beta^{\prime}$ are two deformation parameters which are assumed positive $\left(\beta, \beta^{\prime} \geq 0\right)$. In terms of length $(\mathrm{L})$, mass $(\mathrm{M})$, and time $(\mathrm{T})$ the deformation parameters $\beta$ and $\beta^{\prime}$ have the same dimensions $M^{-2} L^{-2} T^{2}$. Also, $X^{\mu}$ and $P^{\mu}$ are deformed position and momentum operators and $g_{\mu \nu}=g^{\mu \nu}=\operatorname{diag}(1,-1,-1,-1)$. By using Eq. (2) and Schwarz inequality the uncertainty relation for position and momentum by assuming that $\triangle P^{i}$ is isotropic $\left(\triangle P^{i}=\triangle P, \quad i=1,2,3\right)$ becomes

$$
\triangle X^{i} \triangle P \geq \frac{\hbar}{2}\left|1-\beta\left\{\left\langle\left(P^{0}\right)^{2}\right\rangle-3(\triangle P)^{2}-\sum_{j=1}^{3}\left\langle P^{j}\right\rangle^{2}\right\}+\beta^{\prime}\left[(\triangle P)^{2}+\left\langle P^{i}\right\rangle^{2}\right]\right| .
$$

Hence, we arrive at an isotropic absolutely smallest uncertainty in position given by

$$
\left(\triangle X^{i}\right)_{0}=\hbar \sqrt{\left(3 \beta+\beta^{\prime}\right)\left[1-\beta\left\langle\left(P^{0}\right)^{2}\right\rangle\right]}, \quad i \in\{1,2,3\} .
$$

In this study, we consider the special case $\beta^{\prime}=2 \beta$, wherein the position operators $X^{\mu}$ commute to first order in $\beta$. In such a linear approximation, the Lorentz-covariant deformed algebra reads

$$
\begin{gathered}
{\left[X^{\mu}, P^{\nu}\right]=-i \hbar\left(g^{\mu \nu}\left(1-\beta P_{\rho} P^{\rho}\right)-2 \beta P^{\mu} P^{\nu}\right),} \\
{\left[X^{\mu}, X^{\nu}\right]=0,} \\
{\left[P^{\mu}, P^{\nu}\right]=0 .}
\end{gathered}
$$

It is straightforward to verify that the following representations satisfy the Eqs. (7)-(9), at the first order in $\beta$,

$$
\begin{gathered}
X^{\mu}=x^{\mu}, \\
P^{\mu}=\left(1-\beta p^{2}\right) p^{\mu},
\end{gathered}
$$

where $x^{\mu}, p^{\mu}=i \hbar \frac{\partial}{\partial x_{\mu}}=i \hbar \partial^{\mu}$ are position and momentum operators in ordinary relativistic quantum mechanics, and $p^{2}=p_{\alpha} p^{\alpha}$. The Klein-Gordon equation for a spin-0 particle with rest mass $m$ is [14]

$$
p_{\mu} p^{\mu} \Phi-m^{2} c^{2} \Phi=0 .
$$

In particle physics the real Klein-Gordon field describes electrically neutral spin-0 particles such as $\pi^{0}-$ meson. Now the Klein-Gordon equation (12) should be written in generalized form. For this purpose, the momentum operator $p^{\mu}$ must be replaced by deformed momentum operator $P^{\mu}$ from Eq. (11), hence we have

$$
\left(1-\beta p^{2}\right) p_{\mu}\left(1-\beta p^{2}\right) p^{\mu} \Phi-m^{2} c^{2} \Phi=0 .
$$

Neglecting terms of order $\beta^{2}$, the generalized Klein-Gordon equation (13) takes the form

$$
\square \Phi+2 \beta \hbar^{2} \square \square \Phi+\left(\frac{m c}{\hbar}\right)^{2} \Phi=0,
$$


where $\square \equiv \partial_{\mu} \partial^{\mu}$ is the d'Alembertian operator. The term $2 \beta \hbar^{2} \square \square \Phi$ in Eq. (14) can be considered as a quantum gravitational correction. The wave equation (14) is a fourth-order relativistic wave equation that in the limit of $\beta \rightarrow 0$ turns in to the ordinary Klein-Gordon equation. In appendix A we have studied the Lagrangian formulation of the real KleinGordon field in the presence of a minimal length.

\section{Plane-Wave Solutions of the Generalized Klein-Gordon Equation}

In this section, we will obtain the plane-wave solutions of the generalized Klein-Gordon equation (14). We try to find a plane-wave solution of (14):

$$
\Phi=A e^{-i k \cdot x}
$$

where $A \neq 0$ is a complex constant. Equation (15) is a solution of (14) if

$$
2 \beta \hbar^{2}\left(k^{2}\right)^{2}-k^{2}+\left(\frac{m c}{\hbar}\right)^{2}=0
$$

where

$$
k^{2}=k_{\mu} k^{\mu}=\left(\frac{\omega}{c}\right)^{2}-\vec{k} . \vec{k} .
$$

By solving Eq. (16) with respect to $k^{2}$, we obtain two different values for $k^{2}$ as

$$
\begin{aligned}
& k_{+}^{2}=\left(\frac{m_{+} c}{\hbar}\right)^{2}, \\
& k_{-}^{2}=\left(\frac{m_{-} c}{\hbar}\right)^{2},
\end{aligned}
$$

where the non-degenerate effective masses $m_{+}$and $m_{-}$are defined as

$$
\begin{aligned}
& m_{+}=\frac{(1+2 \sqrt{2 \beta} m c)^{\frac{1}{2}}+(1-2 \sqrt{2 \beta} m c)^{\frac{1}{2}}}{2 \sqrt{2 \beta} c}, \\
& m_{-}=\frac{(1+2 \sqrt{2 \beta} m c)^{\frac{1}{2}}-(1-2 \sqrt{2 \beta} m c)^{\frac{1}{2}}}{2 \sqrt{2 \beta} c} .
\end{aligned}
$$

From the standpoint of quantum mechanics, Eqs. (20) and (21) indicate that our field is associated with particles having the effective masses $m_{+}$and $m_{-}$. To avoid particles of complex mass, Eqs. (20) and (21) require that

$$
\beta<\frac{1}{8 m^{2} c^{2}}
$$


Note that at $\beta=\frac{1}{8 m^{2} c^{2}}$ both effective masses are equal, i.e., $m_{+}=m_{-}=m \sqrt{2}$. Using Eqs. (17)-(19) we arrive at the following generalized energy-momentum relations

$$
\begin{aligned}
& E_{p}^{(+) 2}=m_{+}^{2} c^{4}+c^{2}|\vec{p}|^{2}, \\
& E_{p}^{(-) 2}=m_{-}^{2} c^{4}+c^{2}|\vec{p}|^{2},
\end{aligned}
$$

where $E_{p}^{( \pm)}=\hbar \omega_{k}^{( \pm)}$. The effective masses $m_{+}$and $m_{-}$in Eqs. (20) and (21) in the first order over deformation parameter $\beta$ can be written as

$$
\begin{gathered}
m_{+}=\frac{1}{\sqrt{2 \beta} c}-\frac{m^{2}}{2} \sqrt{2 \beta} c \\
m_{-}=m+\beta m^{3} c^{2} .
\end{gathered}
$$

Hence for $\beta \rightarrow 0$ the effective mass $m_{-}$in Eq. (26) reduces to the ordinary mass $m$. Inserting (26) into the equation (24) we find the following generalization for energy-momentum relation

$$
E_{p}^{(-) 2}=m^{2} c^{4}+c^{2}|\vec{p}|^{2}+2 \beta m^{4} c^{6}
$$

When $\beta=0$, equation (27) will be converted into the well-known Einstein energy-momentum relation in the special relativity. On the other hand, the effective mass $m_{+}$in Eq. (25) diverges for small $\beta$. In appendix $\mathrm{B}$, we have shown that the real Klein-Gordon field in the presence of a minimal length possesses one physical state with the effective mass $m_{-}$, and one Weyl ghost with the effective mass $m_{+}$and hence the other generalized energy-momentum relation for the effective mass $m_{+}$in Eq. (23) is entirely new and for $\beta=0$, it has no analog in the special theory of relativity. The general solution of Eq. (14) is a superposition of plane-waves as

$$
\begin{aligned}
\Phi(x) & =\sum_{\vec{k}}\left(\frac{\hbar c^{2}}{2 V \omega_{k}^{(-)}}\right)^{\frac{1}{2}}\left[a(\vec{k}) \exp \left(-\frac{i}{\hbar}\left(E_{p}^{(-)} t-\vec{p} \cdot \vec{r}\right)\right)+a^{\dagger}(\vec{k}) \exp \left(\frac{i}{\hbar}\left(E_{p}^{(-)} t-\vec{p} \cdot \vec{r}\right)\right)\right] \\
& +\sum_{\vec{k}}\left(\frac{\hbar c^{2}}{2 V \omega_{k}^{(+)}}\right)^{\frac{1}{2}}\left[b(\vec{k}) \exp \left(-\frac{i}{\hbar}\left(E_{p}^{(+)} t-\vec{p} \cdot \vec{r}\right)\right)+b^{\dagger}(\vec{k}) \exp \left(\frac{i}{\hbar}\left(E_{p}^{(+)} t-\vec{p} \cdot \vec{r}\right)\right)\right]
\end{aligned}
$$

where we shall take the solutions $\Phi(x)$ to lie in a large cube of side $L$ and volume $V=L^{3}$. The first two terms on the right-hand side of Eq. (28) for $\beta \rightarrow 0$ will be converted to the general solution of the ordinary Klein-Gordon equation [15], while the last two terms on the right-hand side of Eq. (28) for $\beta \rightarrow 0$ are entirely new and they have no analog in ordinary Klein-Gordon theory. By putting $\beta^{\prime}=2 \beta$ into equation (6) and neglecting terms of order $\beta^{2}$, the isotropic minimal length becomes $\left(\triangle X^{i}\right)_{0} \simeq \hbar \sqrt{5 \beta}$. The upper bound for deformation parameter $\beta$ together with isotropic minimal length $\left(\triangle X^{i}\right)_{0}$ are given in Table 1 for some neutral mesons according to Eq. (22). 
Table 1: The upper bound values for deformation parameter $\beta$ and its corresponding isotropic minimal length $\left(\triangle X^{i}\right)_{0}$ (the meson masses are taken from Ref.[16])

\begin{tabular}{|c||c|c|c|c|}
\hline Meson & Quark content & $\begin{array}{c}\text { Mass } \\
\left(M e V / c^{2}\right)\end{array}$ & $\begin{array}{c}\beta_{\text {upper-bound }} \simeq \frac{1}{8 m^{2} c^{2}} \\
\left(10^{36} \frac{s^{2}}{k^{2} m^{2}}\right)\end{array}$ & $\begin{array}{c}\left(\triangle X^{i}\right)_{0} \simeq \hbar \sqrt{5 \beta_{\text {upper-bound }}} \\
\left(10^{-16} m\right)\end{array}$ \\
\hline \hline & & & & \\
$\pi^{0}$ & $(u \bar{u}-d \bar{d}) / \sqrt{2}$ & 134.964 & 24.027 & 11.56 \\
$\eta^{\prime}$ & $(u \bar{u}+d \bar{d}-2 s \bar{s}) / \sqrt{6}$ & 548.8 & 1.453 & 2.84 \\
$\eta^{\prime}$ & $(u \bar{u}+d \bar{d}+s \bar{s}) / \sqrt{3}$ & 957.6 & 0.477 & 1.63 \\
$\eta_{c}$ & $c \bar{c}$ & 2981 & 0.049 & 0.52 \\
\hline
\end{tabular}

\section{Conclusions}

"In the past few years, a large amount of research work has been devoted to the study of the minimal length uncertainty relation. The idea behind this minimal length uncertainty is, if we take into account the effects of quantum fluctuations of the gravitational field in order to incorporate gravity into quantum mechanics, a significant consequence deduced from this unification is that in quantum gravity, there exists a minimal observable length of the order of the Planck distance. This minimal length uncertainty is related to a modification of the standard Heisenberg algebra by adding small corrections to the canonical commutation relations [17]." An immediate consequence of the minimal length uncertainty relation is a generalization of momentum operator according to Eq. (11). This generalized form of momentum operator leads to a fourth-order Klein-Gordon equation. We have shown that our modified Klein-Gordon equation which contains fourth-order derivative of the wave function describes two massive particles, one physical particle with the effective mass $m_{-}$and the other a ghost particle with the effective mass $m_{+}$according to Eqs. (20) and (21). From Eqs. (20) and (21), one finds the restriction on the deformation parameter $\beta: \beta<\frac{1}{8 m^{2} c^{2}}$ which leads to the isotropic minimal length $\left(\triangle X^{i}\right)_{0} \simeq \frac{\sqrt{10}}{4} \frac{\hbar}{m c}$. According to Table 1 the isotropic minimal length in our analysis lies in the interval $10^{-17} m<\left(\triangle X^{i}\right)_{0}<10^{-15} \mathrm{~m}$. The above range for the isotropic minimal length is compatible with the results of Refs. [8-13]. In [812] considering the Lamb shift the authors estimated $\left(\triangle X^{i}\right)_{0} \leq 10^{-16}-10^{-17} \mathrm{~m}$, analysis of electron motion in a Penning trap also gives $\left(\triangle X^{i}\right)_{0} \leq 10^{-16} m$ [13]. On the other hand, consideration of neutron motion in the gravitational field [18] gives large $\left(\triangle X^{i}\right)_{0} \leq 2.4 \times 10^{-9} \mathrm{~m}$ due to significant experimental errors. In Ref. [19], the author studies the standard Casimir effect of two perfectly conducting plates and obtains $\left(\triangle X^{i}\right)_{0} \leq 15 \mathrm{~nm}$. In Ref. [20], the effect of minimal length in the Casimir-Polder interactions between neutral atoms is studied and the author estimates the minimal length must be in the range $80 \mathrm{~nm}<\left(\triangle X^{i}\right)_{0}<10 \mu \mathrm{m}$. Note that the reported values for the isotropic minimal length in Refs. [18-20] are slightly 
different from the numerical values of $\left(\triangle X^{i}\right)_{0}$ in our work and Refs. [8-13].

\section{Acknowledgments}

We would like to thank Ali Asghar Vafainia for discussions during early stages of this work.

\section{Appendix A. Lagrangian Formulation of the Real Klein- Gordon Field in the Presence of a Minimal Length}

The Klein-Gordon Lagrangian density for a real scalar field is [16]

$$
\mathcal{L}\left(\Phi, \partial_{\mu} \Phi\right)=\frac{1}{2} g^{\mu \nu}\left(\partial_{\mu} \Phi\right)\left(\partial_{\nu} \Phi\right)-\frac{1}{2}\left(\frac{m c}{\hbar}\right)^{2} \Phi^{2} .
$$

The Euler-Lagrange equation for the field $\Phi$ is

$$
\frac{\partial \mathcal{L}}{\partial \Phi}-\partial_{\mu}\left(\frac{\partial \mathcal{L}}{\partial\left(\partial_{\mu} \Phi\right)}\right)=0
$$

If we substitute the Lagrangian density (A.1) into the Euler-Lagrange equation (A.2), we will obtain the Klein-Gordon equation as follows

$$
\square \Phi+\left(\frac{m c}{\hbar}\right)^{2} \Phi=0 .
$$

So far we have considered Lagrangians which were functions of field quantities and their first derivatives only. Now we want to obtain the Lagrangian density for the real Klein-Gordon field in the presence of a minimal length. For such a purpose, let us write the Lagrangian density by using the representations (10) and (11), i.e.,

$$
\begin{gathered}
x^{\mu} \rightarrow x^{\mu}, \\
\partial^{\mu} \rightarrow\left(1+\beta \hbar^{2} \square\right) \partial^{\mu} .
\end{gathered}
$$

The result reads

$$
\begin{aligned}
\mathcal{L}= & \frac{1}{2} g^{\mu \nu}\left[\left(1+\beta \hbar^{2} \square\right) \partial_{\mu}\right] \Phi\left[\left(1+\beta \hbar^{2} \square\right) \partial_{\nu}\right] \Phi-\frac{1}{2}\left(\frac{m c}{\hbar}\right)^{2} \Phi^{2} \\
= & \frac{1}{2}\left[g^{\mu \nu}\left(\partial_{\mu} \Phi\right)\left(\partial_{\nu} \Phi\right)-2 \beta \hbar^{2}(\square \Phi)(\square \Phi)\right]-\frac{1}{2}\left(\frac{m c}{\hbar}\right)^{2} \Phi^{2} \\
& +\partial^{\mu}\left[\beta \hbar^{2}\left(\partial_{\mu} \Phi\right)(\square \Phi)\right]+\mathcal{O}\left(\beta^{2}\right) .
\end{aligned}
$$

After neglecting terms of order $\beta^{2}$ and dropping out the total derivative term $\partial^{\mu}\left[\beta \hbar^{2}\left(\partial_{\mu} \Phi\right)(\square \Phi)\right]$ , the Lagrangian density (A.6) will be equivalent to the following Lagrangian density

$$
\mathcal{L}=\frac{1}{2}\left[g^{\mu \nu}\left(\partial_{\mu} \Phi\right)\left(\partial_{\nu} \Phi\right)-2 \beta \hbar^{2}(\square \Phi)(\square \Phi)\right]-\frac{1}{2}\left(\frac{m c}{\hbar}\right)^{2} \Phi^{2} .
$$


The second term on the right-hand side of (A.7) shows the effects of quantum gravitational corrections. If Lagrangian density $\mathcal{L}$ depends on first- and second-order derivatives of the fields, the Euler-Lagrange equations will take the form [21]

$$
\frac{\partial \mathcal{L}}{\partial \Phi}-\partial_{\mu}\left(\frac{\partial \mathcal{L}}{\partial\left(\partial_{\mu} \Phi\right)}\right)+\partial_{\mu} \partial_{\nu}\left(\frac{\partial \mathcal{L}}{\partial\left(\partial_{\mu} \partial_{\nu} \Phi\right)}\right)=0 .
$$

If we substitute the Lagrangian density (A.7) into the generalized Euler-Lagrange equation (A.8), we will obtain the Klein-Gordon equation in the presence of a minimal length as follows

$$
\square \Phi+2 \beta \hbar^{2} \square \square \Phi+\left(\frac{m c}{\hbar}\right)^{2} \Phi=0 .
$$

\section{Appendix B. Feynman Propagator for the Real Klein- Gordon Field in the Presence of a Minimal Length}

In order to discuss the Green's functions of the theory we let the generalized Klein-Gordon field interact with an external source by adding the term $J(x)$ to the right-hand side in Eq. (14). The inhomogeneous generalized Klein-Gordon equation becomes

$$
\left(\square+2 \beta \hbar^{2} \square \square+\left(\frac{m c}{\hbar}\right)^{2}\right) \Phi(x)=J(x) .
$$

Then the solution to the wave equation (B.1) is given by

$$
\Phi(x)=\Phi_{0}(x)-\int \Delta_{F}^{M}(x-y) J(y) d^{4} y,
$$

where $\Delta_{F}^{M}(x-y)$ is the modified Feynman propagator defined by

$$
\left(\square_{x}+2 \beta \hbar^{2} \square_{x} \square_{x}+\left(\frac{m c}{\hbar}\right)^{2}\right) \Delta_{F}^{M}(x-y)=-\delta^{4}(x-y),
$$

and $\Phi_{0}(x)$ is any function that satisfies the homogeneous wave equation

$$
\left(\square+2 \beta \hbar^{2} \square \square+\left(\frac{m c}{\hbar}\right)^{2}\right) \Phi_{0}(x)=0 .
$$

On making the Fourier transform,

$$
\Delta_{F}^{M}(x-y)=\frac{1}{(2 \pi)^{4}} \int d^{4} k D_{F}^{M}(k) e^{-i k \cdot(x-y)},
$$

where $D_{F}^{M}(k)$ is the modified momentum space propagator and substituting it into (B.3), we get

$$
D_{F}^{M}(k)=\frac{1}{k^{2}-2 \beta \hbar^{2}\left(k^{2}\right)^{2}-\left(\frac{m c}{\hbar}\right)^{2}}
$$




$$
\begin{aligned}
& =\frac{1}{2 \beta c^{2}\left(m_{+}^{2}-m_{-}^{2}\right)}\left[\frac{1}{k^{2}-\left(\frac{m_{-} c}{\hbar}\right)^{2}}-\frac{1}{k^{2}-\left(\frac{m_{+} c}{\hbar}\right)^{2}}\right] \\
& =\frac{1}{\left(1-8 \beta m^{2} c^{2}\right)^{\frac{1}{2}}}\left[\frac{1}{k^{2}-\left(\frac{m_{-} c}{\hbar}\right)^{2}}-\frac{1}{k^{2}-\left(\frac{m_{+} c}{\hbar}\right)^{2}}\right] .
\end{aligned}
$$

Equation (B.6) is valid only in the case $m_{+} \neq m_{-}$or $\beta \neq \frac{1}{8 m^{2} c^{2}}$. According to Eqs. (22) and (B.6) we have two particles, one with the effective mass $m_{-}$and the other a Weyl ghost of effective mass $m_{+}$. The ghost gives the negative contribution to the energy [22-25] and as a result, the Hamiltonian is not bounded from below. Therefore, in order to formulate the quantum field theory we must introduce indefinite metrics [22-25]. In the limit $\beta \rightarrow 0$, the modified momentum space propagator $D_{F}^{M}(k)$ in (B.6) smoothly becomes the conventional momentum space propagator $D_{F}(k)[15]$, i.e.,

$$
\begin{aligned}
\lim _{\beta \rightarrow 0} D_{F}^{M}(k) & =D_{F}(k) \\
& =\frac{1}{k^{2}-\left(\frac{m c}{\hbar}\right)^{2}} .
\end{aligned}
$$

\section{References}

[1] S. Capozziello, G. Lambiase and G. Scarpetta, Int. J. Theor. Phys. 39, 15 (2000); L. Xiang, Commun. Theor. Phys. 48, 93 (2007); J. sadeghi, J. Math. Phys. 48, 113508 (2007); B. Vakili, Phys. Rev. D 77, 044023 (2008).

[2] C. Quesne and V. M. Tkachuk, J. Phys. A: Math. Gen. 39, 10909 (2006).

[3] C. Quesne and V. M. Tkachuk, Czech. J. Phys. 56, 1269 (2006).

[4] D. Bouaziz and M. Bawin, Phys. Rev. A 76, 032112 (2007).

[5] B. Bolen and M. Cavaglia, Gen. Rel. Grav. 37, 1255 (2005); M. R. Setare, Phys. Rev. D 70, 087501 (2004); M. R. Setare, Int. J. Mod. Phys. A 21, 1325 (2006).

[6] V. M. Tkachuk, Journal of Physical Studies 11, 41 (2007).

[7] A. Kempf, G. Mangano and R. B. Mann, Phys. Rev. D 52, 1108 (1995).

[8] S. Das and E. C. Vagenas, Phys. Rev. Lett. 101, 221301 (2008); S. Das and E. C. Vagenas, Can. J. Phys. 87, 233 (2009).

[9] F. Brau, J. Phys. A: Math. Gen. 32, 7691 (1999).

[10] S. Benczik, L. N. Chang, D. Minic and T. Takeuchi, Phys. Rev. A 72, 012104 (2005).

[11] M. M. Stetsko and V. M. Tkachuk, Phys. Rev. A 74, 012101 (2006). 
[12] M. M. Stetsko, Phys. Rev. A 74, 062105 (2006).

[13] L. N. Chang, D. Minic, N. Okamura and T. Takeuchi, Phys. Rev. D 65, 125027 (2002).

[14] W. Greiner, Relativistic Quantum Mechanics: Wave Equations, Third Edition (Springer-Verlag, Berlin, 2000).

[15] F. Mandl and G. Shaw, Quantum Field Theory (John Wiley \& Sons, 1984).

[16] D. Griffiths, Introduction to Elementary Particles (John Wiley \& Sons, 1987).

[17] M. Falek and M. Merad, J. Math. Phys. 50, 023508 (2009).

[18] F. Brau and F. Buisseret, Phys. Rev. D 74, 036002 (2006).

[19] Kh. Nouicer, J. Phys. A: Math. Gen. 38, 10027 (2005).

[20] O. Panella, Phys. Rev. D 76, 045012 (2007).

[21] A. O. Barut, Electrodynamics and Classical Theory of Fields and Particles (Dover, New York, 1980).

[22] S. W. Hawking and T. Hertog, Phys. Rev. D 65, 103515 (2002).

[23] D. L. Nordstrom, Phys. Rev. D 4, 1611 (1971).

[24] N. H. Barth and S. M. Christensen, Phys. Rev. D 28, 1876 (1983).

[25] S. I. Kruglov, Can. J. Phys. 85, 887 (2007). 